\title{
A call for collaboration
}

\section{Specialization has its place, but truly innovative advances in HIV research usually come from interdisciplinary efforts, reports Unmesh Kher.}

$\mathrm{t}$ is probably a good thing Rowena Johnston got her doctoral degree in neuroscience.

When Johnston joined the Foundation for AIDS Research (amfAR) nearly nine years ago, most people working on HIV would have felt foolish even mentioning a cure. As an outsider, however, she could look at the proposition with fresh eyes.

"I wasn't loaded down with all the baggage that was preventing people from explicitly pursuing a cure," recalls Johnston, who became amfAR's director of research in 2004. Her unabashed ambition has now found expression: in March 2010, amfAR requested proposals for a consortium dedicated to exploring "the barriers to and potential for HIV eradication." In May 2010, it issued grants totalling US $\$ 1.2$ million to launch the initiative.

The rest of the field is eyeing the same goal. A paper in Science last year sketched out the organizational structure of a 'collaboratory' for a cure. The US National Institutes of Allergy and Infectious Diseases (NIAID) is preparing a request for applications explicitly aimed at exploring
"What we need is for people from other fields to work on the problem." from a conviction shared by many in the field that their profession has become at once too big and too internally ghettoized.

"There are more [HIV] investigators than there are nucleotides in the HIV genome," says Douglas Richman, a professor of pathology and medicine at the University of California, San Diego, and lead author on the Science paper. "It shows you just how clever the virus is."

\section{Isolated experts}

Excessive specialization has also hampered the flow of information between researchers. For example, HIV immunologists rarely interact with immunologists in other fields, notes Bruce Walker, professor of medicine at Harvard Medical School, and director of the US $\$ 100$ million Ragon Institute, launched last year in Massachusetts with an explicitly how HIV hides out and persists in the body, and for developing safe strategies to eliminate the latent virus.

These schemes have more in common than just their aims. They also share a view of how best to attain them: through an interdisciplinary team of scientists with diverse backgrounds.

amfAR has awarded the bulk of its funding to three collaborative teams that will each address distinct aspects of the problem. These groups are expected to talk frequently and, where helpful, participate in each other's projects. NIAID's programme would also seek to support a collaborative effort that includes teams from academia, the private sector and the US National Institutes of Health - much as was envisioned in the Science paper.

A glance at HIV/AIDS research suggests that interdisciplinary groups are increasingly the order of the day. Such groups have emerged interdisciplinary mission.

Because there is no mouse model for AIDS, Walker adds, those who develop HIV vaccines do not interact with immunologists who work with mice, and miss out on valuable insights. "I think there are basic universal truths that can be generated in mouse models and applied to humans," he says.

If history is any guide, bridging some of these gaps would be wise. Most major breakthroughs in HIV research have relied on the integrated contributions of multiple disciplines. The discovery of HIV depended on the combined efforts, on two continents, of clinicians treating inexplicably immunecompromised patients, epidemiologists tracking the emerging disease and virologists racing to isolate its causative agent.

Likewise, when the HIV genome was sequenced in 1984, the possibility of attacking it with drugs less toxic and more effective than the first chemotherapeutic agent AZT (zidovudine) drew a host of new scientists into the fray, not least structural biologists. The subsequent determination of HIV protease structures led to the development of inhibitors of the enzyme,

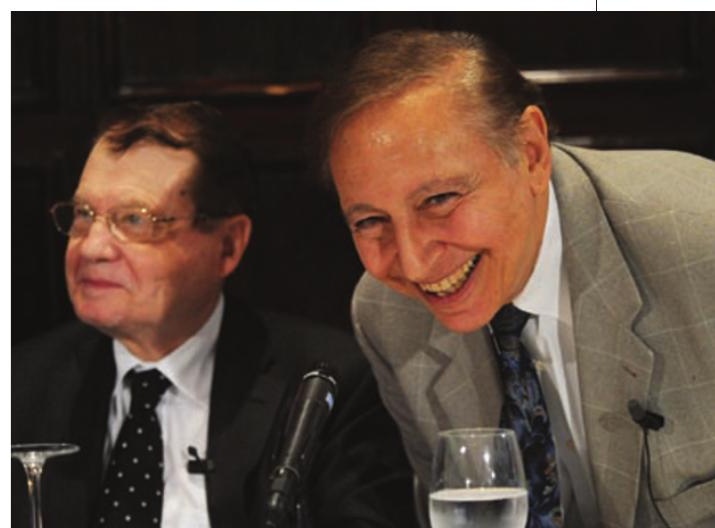

The discovery of HIV by France's Luc Montagnier (left) and American Robert Gallo (right) depended on the combined efforts of clinicians, epidemiologists and virologists.

which was a landmark in rational drug design and primer to a cascade of antiretroviral drug discovery.

"Perhaps the biggest breakthrough of all," says Anthony Fauci, director of NIAID, "has been the successful development of now more than 30 drugs that, in combination, have totally transformed the lives of HIV-positive individuals."

As the field evolved, the number of contributing disciplines multiplied. The development of sensitive assays to quantify HIV in the bloodstream - the typically unsung contribution of analytical biochemistry - touched off an avalanche of discovery in the 1990s.

"The mere development of these assays allowed the field to understand the correlation between viral load and disease progression," recalls David Ho, chief executive officer of the Aaron Diamond AIDS Research Center in New York City.

Ho's work captured the interplay of the virus and its target cells, and linked those dynamics to disease progression. Mathematics and computer modelling played an integral role in those studies, which Ho notes was unusual in virology at the time. His work led to the standard use of drug cocktails to treat AIDS.

\section{Fresh blood}

Today, HIV researchers generally agree that the two areas most in need of interdisciplinary efforts are the quest for an AIDS vaccine and the search for a cure. "Those of us who've been in the field haven't solved the problem," says Walker. "What we need is for people from other fields to work on the problem."

Innately interdisciplinary approaches such as systems biology could shed light on some of the toughest problems facing the field, such as how activating certain receptors on cells of the innate immune system - the generic first line of defence - influences the adaptive 


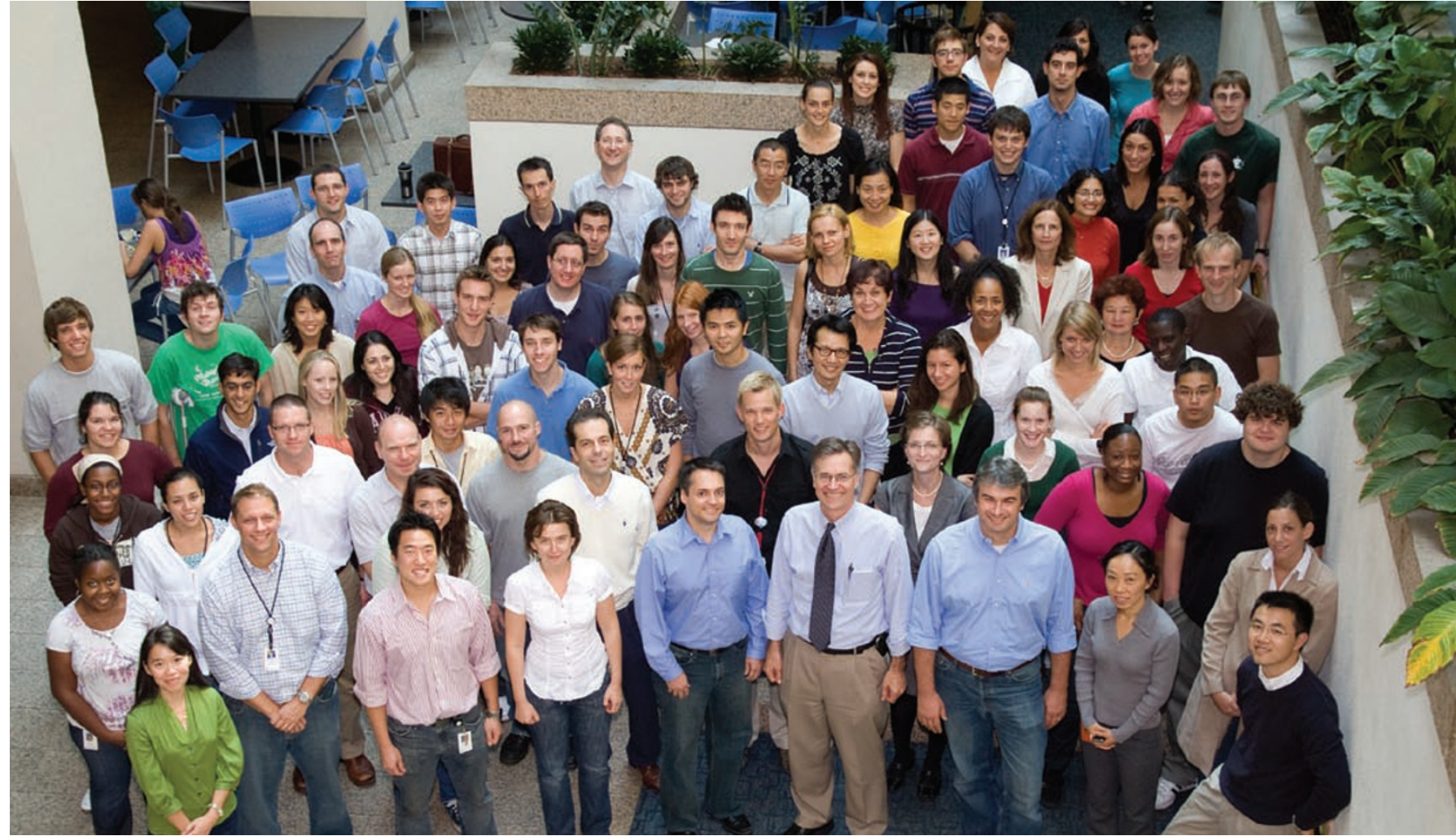

The US $\$ 100$ million Ragon Institute, launched last year, employs engineers, clinicians, basic immunologists, computational biologists and geneticists.

immune response to HIV infection, which is dominated by antibody and T-cell responses against specific pathogens or antigens.

"There has been a failure to appreciate what kinds of immune responses are needed to protect against HIV," notes Bali Pulendran, professor of pathology and laboratory medicine at Emory University. "It's a larger issue than just HIV research because the way people have analysed immune responses in the context of any disease, or any antigen, is through the use of standard immune assays."

In 2008, Pulendran applied a systemsbiology approach to predict - with high accuracy - the precise immune responses elicited by the powerful yellow fever vaccine. Using a DNA microarray, Pulendran and colleagues measured changes in gene expression in response to the vaccine and then used that expression profile to estimate levels of the subsequent $\mathrm{T}$-cell and $\mathrm{B}$-cell responses.

Similar approaches relying on microarray analysis, proteomics, microRNA profiling and other techniques generate thousands of variables for computational analysis, and could be applied together to solve seemingly intractable problems, says Pulendran. "In the past four or five years or so," he says, "people have begun to understand that to fully capture the molecular and cellular mechanisms of immunity, you need a much more global approach."

\section{Vaccine wars}

Most agree that interdisciplinary problemsolving is a good thing. More controversial are the consortia for HIV vaccine research established by two of the field's major funders.
In 2005, the US National Institutes of Health (NIH) established a US $\$ 315$ million, sevenyear venture named the Center for HIV/AIDS Vaccine Immunology (CHAVI). It was followed a year later by the Bill \& Melinda Gates Foundation's US\$287 million constellation of consortia named the Coalition for AIDS Vaccine Discovery. Many complain that these initiatives have concentrated scarce funding on a small group of researchers.

"It has a way of homogenizing the field," says Ho, who first raised his concerns when CHAVI was just a concept. "These people are forced to meet - it's almost dictated by the grant funding structure. They talk about the same things over and over, and pretty soon I think everybody thinks the same way, when what we need in the field is more ideas, new ideas."

Instead, say Ho and others, collaborative groups should form naturally. Julie Overbaugh, a virologist at the Fred Hutchinson Cancer Research Center in Seattle, adds that the AIDS vaccine consortia might also be premature. "We're still early in the game, in terms of potential vaccine candidates that might ever make it to the real world," she says. "The 'groupthink' can be a bit of a problem at this point. Dominant personalities can win in those kinds of settings."

Fauci bristles at such criticism, pointing out that CHAVI's director, Barton Haynes of Duke University, has a reputation for openmindedness, and routinely takes advice from many investigators in the field.

"CHAVI implements the ideas of a lot of investigators in a synergistic and collaborative way," Fauci says. He points out that the bulk of funding for HIV vaccines still goes to individual grants. Of the US\$561 million the $\mathrm{NIH}$ set aside for HIV vaccine research in fiscal year 2009, CHAVI's cut was US\$49.8 million. Besides, Fauci says, many of the people funded through CHAVI also have independent investigator grants - underscoring the fact that its members are not group thinkers.

Some say a CHAVI-like consortium is just what the field needs to find a cure for HIV. In the Science paper, Richman and several other leading scientists argued that the NIH should provide "funding, regulatory oversight and coordination" to a multidisciplinary, multisector collaboration - one that depends as much on the contributions of big pharma as those of academia. Richman says he does not believe amfAR's initiative has the resources to fund a true consortium.

Johnston counters that the organization has successfully fostered other underfunded areas of HIV research, including studies on microbicides and the preclinical development of several antiretroviral drug classes. In many cases, she says, amfAR's initial investments were followed by support from other institutions, including the NIH.

The organization hopes to catalyse a collaborative movement towards a cure, says Johnston. "It's probably fair to say that most AIDS researchers are still sceptical that there can ever be a cure," she says. "But our view is that if you don't even try, you can guarantee that there won't ever be one."

Unmesh Kher is a freelance writer in New York City and a consultant for the International AIDS Vaccine Initiative. 